\title{
TROPICAL MEROMORPHIC FUNCTIONS IN A FINITE INTERVAL
}

\author{
Ilpo Laine and Kazuya Tohge \\ University of Eastern Finland, Department of Physics and Mathematics \\ P.O. Box 111, FI-80101 Joensuu, Finland; ilpo.laine@joensuu.fi \\ Kanazawa University, College of Science and Engineering \\ Kakuma-machi, Kanazawa, 920-1192, Japan; tohge@se.kanazawa-u.ac.jp
}

\begin{abstract}
The tropical Nevanlinna theory in the whole real line $\mathbf{R}$ describes value distribution of continuous piecewise linear functions of a real variable with arbitrary real slopes, called tropical meromorphic functions, similarly as value distribution of meromorphic functions of a complex variable is described by the classical Nevanlinna theory in the whole complex plane C. As a tropical counterpart to the Nevanlinna theory in a disc or an annulus centered at the origin, we introduce in this paper a value distribution theory of continuous piecewise linear functions in a symmetric finite open interval $(-R, R)$. The shift operator (difference operator) has a key role in the tropical value distribution theory in $\mathbf{R}$ corresponding to the role of the differential operator in the Nevanlinna theory in a subregion of $\mathbf{C}$. However, the affine shift $x \mapsto x+c$ does not operate properly in finite intervals. Therefore, we introduce a shift $x \mapsto s_{\tau}(x)$ which may be called as the tropical hyperbolic shift. This notion enables us to obtain the quotient estimate $m\left(r, f\left(s_{\tau}(x)\right) \oslash f(x)\right)=o(1) T(r, f)$ for tropical meromorphic functions $f(x)$ defined in an interval $(-R, R)$ in $\mathbf{R}$, corresponding to the logarithmic derivative estimate in the Nevanlinna theory for meromorphic functions $f(z)$ defined in a disc or in an annulus. A sort of the second main theorem is also stated by means of this estimate. Concerning hyperbolic shift and the second main theorem, we assume an order restriction to $f(x)$. This restriction is shown to be necessary by an example.
\end{abstract}

\section{Introduction and notation}

Tropical Nevanlinna theory in the real line, see [5], [7] and [10], describes value distribution of continuous piecewise linear functions of a real variable, similarly as value distribution of meromorphic functions of a complex variable is described by the classical Nevanlinna theory in the complex plane, see [1] and [6].

The original paper [5] on tropical Nevanlinna theory in the real line (restricting to integer slopes) has been subsequently extended in [10] to include tropical counterpart to the second main theorem (with arbitrary real slopes). A further extension has been recently made in [8] to considering tropical holomorphic curves in a tropical projective space. In these preceding papers, tropical Nevanlinna theory has been treated in the global setting. We are now considering the theory in the local sense by going to treat tropical meromorphic functions and their Nevanlinna theory in a finite interval.

https://doi.org/10.5186/aasfm.2019.4418

2010 Mathematics Subject Classification: Primary 39A12; Secondary 30D35, 39A13.

Key words: Difference equation, tropical meromorphic function, tropical Nevanlinna theory.

The first author has been supported by the Academy of Finland grant 286877.

The work of the second author has been supported by JSPS KAKENHI Grant numbers 16K05194, 25400131 and 22540181. 
Recalling the standard one-dimensional tropical framework, we shall consider a max-plus semi-ring endowing $\mathbf{R}_{-\infty}:=\mathbf{R} \cup\{-\infty\}$ with tropical addition

$$
x \oplus y:=\max (x, y)
$$

and tropical multiplication

$$
x \otimes y:=x+y .
$$

We also use the notations $x \oslash y:=x-y$ and $x^{\otimes \alpha}:=\alpha x$ for $\alpha \in \mathbf{R}$. The neutral elements for the tropical operations are $0_{\circ}=-\infty$ for addition and $1_{\circ}=0$ for multiplication. We note that $1_{\diamond} \oplus x=\max (x, 0)$ and $0_{\circ} \otimes x=0$ 。 for any $x \in \mathbf{R}$. We sometimes denote $\max (x, 0)$ by $x^{+}$. Observe that such a structure is not a ring, since not all elements have tropical additive inverses. For a general background concerning tropical mathematics, see [11], for example.

In this note, we introduce Nevanlinna theory of tropical meromorphic functions in an open interval $(-R, R), 0<R<+\infty$. Here we give the reason why one may concentrate on such a symmetric interval. Suppose that $F(x)$ is tropical meromorphic in a general open finite interval $\left(R_{1}, R_{2}\right)$ with $R_{1}, R_{2} \in \mathbf{R}$. Then, instead of the pair of the function $F(x)$ and the interval $\left(R_{1}, R_{2}\right)$, we can simply consider the pair of the function $f(x)$ and the interval $(-R, R)$ given by

$$
f(x)=F\left(x+\frac{R_{1}+R_{2}}{2}\right) \quad \text { and } \quad R=\frac{R_{2}-R_{1}}{2} .
$$

Further, we may also apply our theory to tropical meromorphic functions $F(x)$ in a half -infinite interval, say in $\left(-\infty, R_{2}\right)$ with $R_{2} \in \mathbf{R}$. Then there exists an $R_{1} \in$ $\left(-\infty, R_{2}\right)$ such that the function $F(x)$ is linear, $\ell(x)$ say, in a neighborhood of $x=R_{1}$. Then consider two tropical meromorphic functions $F_{1}(x)$ (defined in the whole $\mathbf{R}$ ) and $F_{2}(x)$ (defined in the finite interval $\left.\left(R_{1}, R_{2}\right)\right)$ as follows:

$$
F_{1}(x)= \begin{cases}F(x) & \left(-\infty<x \leq R_{1}\right) \\ \ell(x) & \left(R_{1}<x<+\infty\right)\end{cases}
$$

and

$$
F_{2}(x)=F(x) \quad\left(R_{1}<x<R_{2}\right) .
$$

Then we may study value properties of $F(x)$ in the half-infinite closed interval $\left(-\infty, R_{1}\right]$ through those of $F_{1}(x)$ by means of the tropical Nevanlinna theory on $\mathbf{R}$ as the value distribution of $F_{1}(x)=\ell(x)$ in the half-infinite interval $\left(R_{1}, \infty\right)$ is trivial. On the other hand, value properties of $F(x)=F_{2}(x)$ in the open interval $\left(R_{1}, R_{2}\right)$ follow by means of the tropical Nevanlinna theory to be introduced in this note. Similarly one may consider half-infinite intervals $\left(R_{1},+\infty\right)$ with $R_{1} \in \mathbf{R}$.

Returning to the study of tropical value distribution in a finite interval $\left(R_{1}, R_{2}\right)$, we are concerned with the case when our function $F(x)$ can be continued beyond neither of the endpoints to a tropical meromorphic function in any larger interval. Then the endpoints $x=R_{1}$ and $x=R_{2}$ may be considered as essential singularities of the tropical meromorphic function $F(x)$ and we may say that $F(x)$ is transcendental near $x=R_{1}$ and $x=R_{2}$. In fact, this happens when the set of all the roots and poles of $F(x)$ (see Definition 1.3 below) has both of the endpoints as its limit points.

Thus, from now on, we are considering transcendental tropical meromorphic functions in an open interval $(-R, R), 0<R<+\infty$ and we will study their value distribution by applying the finite interval version of the tropical Nevanlinna theory, to 
be described below. For convenience of the reader, we first restate some of the basic definitions and notations from the real line case:

Definition 1.1. A continuous piecewise linear function $f$ of an interval $(-R, R)$ to $\mathbf{R}$ is said to be tropical meromorphic on $(-R, R)$, where $0<R<+\infty$.

Observe that whenever $f:(-R, R) \rightarrow \mathbf{R}$ is a continuous piecewise linear function, the set of discontinuities of the derivative $f^{\prime}$, see below, might have limit points possibly at $x=R$, at $x=-R$ or at both of $x= \pm R$.

Definition 1.2. A point $x$ of derivative discontinuity of a tropical meromorphic function $f$ such that

$$
\omega_{f}(x):=\lim _{\varepsilon \rightarrow 0+}\left(f^{\prime}(x+\varepsilon)-f^{\prime}(x-\varepsilon)\right)<0
$$

is said to be a pole of $f$ of multiplicity $-\omega_{f}(x)$, while if $\omega_{f}(x)>0$, then the point $x$ is called a root (or a zero-point) of $f$ of multiplicity $\omega_{f}(x)$.

Observe that the multiplicity $\left|\omega_{f}(x)\right|$ may be any real number, to be denoted as $\tau_{f}(x)$ in what follows.

Definition 1.3. If a tropical meromorphic function $f$ in the interval $(-R, R)$ has only finitely many poles and roots in $(-R, R)$ implying that $f$ is continuous and piecewise linear in the closed interval $[-R, R]$, then we may call it tropical meromorphic of rational type in $(-R, R)$. Otherwise, we say that $f$ is transcendental in $(-R, R)$, meaning that it cannot be defined at both of the points $x= \pm R$.

Suppose, in what follows, that $f$ is tropical meromorphic in an interval $(-R, R)$ for a fixed number $R>0$. The Nevanlinna functions are now easily set up similarly as in [5], [7] and [10]:

Definition 1.4. The tropical proximity function for tropical meromorphic functions $f$ in the interval $(-R, R)$ is defined as

$$
m(r, f):=\frac{f(-r)^{+}+f(r)^{+}}{2},
$$

where $f(x)^{+}:=\max \{f(x), 0\}$.

Next, we define tropical counting functions.

Definition 1.5. We denote by $n(t, f)$ the number of distinct poles of $f$ in the symmetric interval $(-t, t)$ for $0<t<R$, each pole $b_{\nu} \in(-t, t), 1 \leq \nu \leq N$ contributing to $n(r, f)$ by its multiplicity $\tau_{f}\left(b_{\nu}\right)$. Then the tropical counting function for the poles in $(-R, R)$ is defined as

$$
\begin{aligned}
N(r, f) & :=\frac{1}{2} \int_{0}^{r} n(t, f) d t=\frac{1}{2} \sum_{\nu=1}^{N} \tau_{f}\left(b_{\nu}\right)\left(r-\left|b_{\nu}\right|\right), \\
& =\frac{1}{2 r} \sum_{\nu=1}^{N} \tau_{f}\left(b_{\nu}\right)\left(r-\max \left(b_{\nu}, 0\right)\right)\left(\min \left(b_{\nu}, 0\right)+r\right)
\end{aligned}
$$

for any $0<r<R$.

Note that the weight $(r-t)$ in the integral representation of $N(r, f)$ drops off in the infinite case $R=\infty$. However, most of the consequences are the same as in the tropical theory in $\mathbf{R}$. 
For the convenience of the reader, we work out the second equality of (1.3). Given $t \in(0, R)$, we put

$$
n_{+}(t, f)=\sum_{0<b_{\nu}<t} \tau_{f}\left(b_{\nu}\right) \text { and } n_{-}(t, f)=\sum_{0<-b_{\nu}<t} \tau_{f}\left(b_{\nu}\right),
$$

and thus we have

$$
n(t, f)=\sum_{\left|b_{\nu}\right|<t} \tau_{f}\left(b_{\nu}\right)=n_{+}(t, f)+n_{-}(t, f)+\tau_{f}(0) .
$$

Using Riemann-Stieltjes integrals and integrating by parts, we obtain

$$
\begin{aligned}
& \sum_{0<b_{\nu}<r} \tau_{f}\left(b_{\nu}\right)\left(r-b_{\nu}\right)=\int_{0}^{r}(r-t) d n_{+}(t, f) \\
& =(r-r) n_{+}(r, f)-(r-0) n_{+}(0, f)-\int_{0}^{r} n_{+}(t, f) d(r-t)=\int_{0}^{r} n_{+}(t, f) d t,
\end{aligned}
$$

and similarly

$$
\sum_{0<-b_{\nu}<r} \tau_{f}\left(b_{\nu}\right)\left(r+b_{\nu}\right)=\int_{0}^{r}(r-t) d n_{-}(t, f)=\int_{0}^{r} n_{-}(t, f) d t .
$$

This implies

$$
\begin{aligned}
& \sum_{\left|b_{\nu}\right|<r} \tau_{f}\left(b_{\nu}\right)\left(r-\left|b_{\nu}\right|\right)=\int_{0}^{r} n_{+}(t, f) d t+\int_{0}^{r} n_{-}(t, f) d t+\tau_{f}(0) r \\
& =\int_{0}^{r}\left\{n_{+}(t, f)+n_{-}(t, f)+\tau_{f}(0)\right\} d t=\int_{0}^{r} n(t, f) d t .
\end{aligned}
$$

Since Definition 1.5 is the same as in the real line case, the next lemma is immediate:

Lemma 1.6. For a given tropical meromorphic function $g(x)$ in $(-R, R)$, the counting function $N(r, g)$ is continuous and non-decreasing with respect to $r$.

In fact, $\frac{d}{d r} N(r, g) \geq 0$ holds for any $r \in[0, R)$, since by definition and a simple observation, we have

$$
\frac{d}{d r} N(r, g)=\frac{d}{d r}\left\{\frac{1}{2} \int_{0}^{r} n(t, g) d t\right\}=\frac{1}{2} n(r, g) \geq 0 .
$$

Definition 1.7. Define the tropical characteristic function $T(r, f)$ in $(-R, R)$ in the usual way as

$$
T(r, f):=m(r, f)+N(r, f) .
$$

For a tropical meromorphic function $f(x)$ in $(-R, R)$, the tropical Jensen formula

$$
T(r, f)-T(r,-f)=f(0)
$$

holds for $0 \leq r<R$ as in [5], [7] and [10], to be treated in more detail below.

\section{Tropical Poisson-Jensen formula}

In this section, we recall how to express a given tropical meromorphic function in $(-R, R)$ with $0<R \leq \infty$ by means of its zeros (roots) and poles together with multiplicity. 
Theorem 2.1. Suppose $f$ is a tropical meromorphic function in $(-R, R)$ and $R$ is a number satisfying $0<R \leq \infty$. Denote the distinct zeros (roots), respectively poles, of $f$ in this interval $(-r, r) \subset(-R, R)$ by $a_{j}$, respectively by $b_{k}$, with their corresponding multiplicities $\tau_{f}$ attached. Then for any $x \in(-r, r)$ we have the tropical Poisson-Jensen formula

$$
\begin{aligned}
f(x)= & \frac{f(-r)+f(r)}{2}+\frac{f(r)-f(-r)}{2 r} x \\
& -\frac{1}{2 r} \sum_{\left|a_{j}\right|<r} \tau_{f}\left(a_{j}\right)\left(r-\max \left(a_{j}, x\right)\right)\left(\min \left(a_{j}, x\right)+r\right) \\
& +\frac{1}{2 r} \sum_{\left|b_{k}\right|<r} \tau_{f}\left(b_{k}\right)\left(r-\max \left(b_{k}, x\right)\right)\left(\min \left(b_{k}, x\right)+r\right) .
\end{aligned}
$$

In the particular, for $x=0$, we obtain the tropical Jensen formula

$$
f(0)=\frac{f(-r)+f(r)}{2}-\frac{1}{2 r} \sum_{\left|c_{\nu}\right|<r} \omega_{f}\left(c_{\nu}\right)\left(r-\max \left(c_{\nu}, 0\right)\right)\left(\min \left(c_{\nu}, 0\right)+r\right),
$$

where we put $\left\{c_{\nu}\right\}=\left\{a_{j}\right\} \cup\left\{b_{k}\right\}$.

As for the proof of Theorem 2.1, we refer to [7, Theorem 3.1, p. 66] or to [5, Lemma 3.1], where the interval $(-r, r)$ is indeed assumed to be finite.

By using the identity

$$
(r-\max (c, r))(\min (c, x)+r)=r^{2}-|c-x| r-c x,
$$

we have the following result as given in [5] and [10]:

Corollary 2.2. Suppose $f$ is a tropical meromorphic function in $(-R, R)$. Then for any $x \in(-r, r) \subset(-R, R)$ we have

$$
f(x)=\frac{f(r)+f(-r)}{2}+\frac{x}{2 r}\{f(r)-f(-r)\}-\frac{1}{2 r} \sum_{\left|c_{\nu}\right|<r} \omega_{f}\left(c_{\nu}\right)\left(r^{2}-\left|c_{\nu}-x\right| r-c_{\nu} x\right),
$$

and

$$
f(0)=\frac{f(r)+f(-r)}{2}-\frac{1}{2} \sum_{\left|c_{\nu}\right|<r} \omega_{f}\left(c_{\nu}\right)\left(r-\left|c_{\nu}\right|\right),
$$

for the distinct roots and poles $c_{\nu}$ of $f$ in $(-R, R)$.

With tropical Nevanlinna characteristic functions, the latter identity immediately becomes the formula (1.5).

\section{Basic Nevanlinna theory in the tropical setting}

Basic Nevanlinna theory inequalities as proved in [5], [7] and [10] still hold in the interval $(-R, R)$ as well. Here we use the notation $V(r, f)$ as a joint notation for $m(r, f), N(r, f)$ and $T(r, f)$, unless otherwise specified.

Lemma 3.1. Let $f$ and $g$ be tropical meromorphic in the interval $(-R, R)$.

(i) If $f(x) \leq g(x)$ holds for all $x \in(-R, R), m(r, f) \leq m(r, g)$ holds for all $r \in[0, R)$.

(ii) Given a positive real number $\alpha$, we have

$$
V\left(r, f^{\otimes \alpha}\right)=V(r, \alpha f)=\alpha V(r, f)
$$

for all $r \in[0, R)$. 
(iii) For all $r \in[r, R)$,

$$
V(r, f \otimes g)=V(r, f+g) \leq V(r, f)+V(r, g) .
$$

(iv) For all $r \in[r, R)$,

$$
V(r, f \oplus g)=V(r, \max (f, g)) \leq V(r, f)+V(r, g) .
$$

Remark. Observe that whenever $f(x) \leq g(x)$ for all $x \in(-R, R)$, the inequality $N(r, f) \leq N(r, g)$ is not necessarily true. Similarly, the inequality $V(r, f \oplus g) \leq$ $V(r, f) \oplus V(r, g)$, that is,

$$
V(r, \max (f, g)) \leq \max \{V(r, f), V(r, g)\}
$$

may fail for $V=N, T$, as shown by the following simple examples.

On the other hand, (i) shows that we always have

$$
\max \{m(r, f), m(r, g)\} \leq m(r, \max (f, g)) .
$$

Example. (1) Let us consider two functions $f(x), g(x)$ in $(-R, R)$ given by

$$
g(x)= \begin{cases}x+R / 2 & (-R<x \leq 0), \\ -x+R / 2 & (0<x<R)\end{cases}
$$

and

$$
f(x)= \begin{cases}x+R / 2 & (-R<x \leq-R / 4), \\ -x & (-R / 4<x \leq 0), \\ x & (0<x \leq R / 4), \\ -x+R / 2 & (R / 4<x<R) .\end{cases}
$$

Observe that $g(x)$ has only one pole at $x=0$ of multiplicity 2 , while $f(x)$ has two poles of multiplicity 2 at $x= \pm R / 4$. Clearly, $f(x) \leq g(x)$ for all $x \in(-R, R)$. For the counting functions, we have

$$
N(r, f)= \begin{cases}0 & (0 \leq r \leq R / 4), \\ \frac{2(-R / 4+r)+2(r-R / 4)}{2}=2(r-R / 4) & (R / 4<r<R),\end{cases}
$$

$N(r, g)=\frac{2(0+r)}{2}=r(0 \leq r<R)$, and thus

$$
N(r, f)-N(r, g)= \begin{cases}-r & (0 \leq r \leq R / 4) \\ r-R / 2 & (R / 4<r<R) .\end{cases}
$$

Hence

$$
\begin{cases}N(r, f)<N(r, g) & \text { whenever } r \in[0, R / 2) \\ N(r, f)>N(r, g) & \text { whenever } r \in(R / 2, R) .\end{cases}
$$

Moreover, since we have

$$
m(r, f)= \begin{cases}r & (0 \leq r<R / 4) \\ -r+R / 2 & (R / 4 \leq r<R / 2) \\ 0 & (R / 2 \leq r<R)\end{cases}
$$

and

$$
m(r, g)= \begin{cases}-r+R / 2 & (0 \leq r<R / 2) \\ 0 & (R / 2 \leq r<R)\end{cases}
$$


their characteristic functions are estimated as

$$
T(r, f)= \begin{cases}r & (0 \leq r<R / 2) \\ 2(r-R / 4) & (R / 2 \leq r<R)\end{cases}
$$

and

$$
T(r, g)= \begin{cases}R / 2 & (0 \leq r<R / 2) \\ r & (R / 2 \leq r<R) .\end{cases}
$$

Therefore, $T(r, f)-T(r, g)=r-R / 2$ for all $r \in[0, R)$, and so

$$
\begin{cases}T(r, f)<T(r, g) & \text { whenever } r \in[0, R / 2), \\ T(r, f)>T(r, g) & \text { whenever } r \in(R / 2, R) .\end{cases}
$$

(2) We next consider another pair of tropical meromorphic functions given as

$$
f_{1}(x)= \begin{cases}x+R / 2 & (-R<x \leq-R / 4) \\ -x & (-R / 4<x<R)\end{cases}
$$

and

$$
f_{2}(x)= \begin{cases}x & (-R<x \leq R / 4) \\ -x+R / 2 & (R / 4<x<R)\end{cases}
$$

It is easy to see that their tropical sum $f_{1}(x) \oplus f_{2}(x)$ coincides the above $f(x)$ for all $x \in(-R, R)$. In fact, by drawing their graphs, one observes the following relations:

$$
\begin{array}{llrl}
g(x)=f(x)=f_{1}(x)>f_{2}(x) & & (-R<x \leq-R / 4), \\
g(x)>f(x)=f_{1}(x)>f_{2}(x) & & (-R / 4<x<0), \\
g(x)>f(x)=f_{1}(x)=f_{2}(x) & & (x=0), \\
g(x)>f(x)=f_{2}(x)>f_{1}(x) & & (0<x<R / 4), \\
g(x)=f(x)=f_{2}(x)>f_{1}(x) & & (R / 4 \leq x<R) .
\end{array}
$$

It follows by the symmetric relation $f_{2}(x)=f_{1}(-x)$ for all $x \in(-R, R)$ that

$$
N\left(r, f_{1}\right)=N\left(r, f_{2}\right)=\frac{1}{2} N(r, f)= \begin{cases}0 & (0 \leq r \leq R / 4), \\ r-R / 4 & (R / 4<r<R) .\end{cases}
$$

Therefore,

$$
N\left(r, f_{1} \oplus f_{2}\right)=N(r, f)=N\left(r, f_{1}\right)+N\left(r, f_{2}\right)=2 \max \left\{N\left(r, f_{1}\right), N\left(r, f_{2}\right)\right\}
$$

for all $r \in[0, R)$. Of course, this quantity is strictly greater than $\max \left\{N\left(r, f_{1}\right), N\left(r, f_{2}\right)\right\}$ only if $r \in(R / 4, R)$. Similarly, we have $m\left(r, f_{1}\right)=m\left(r, f_{2}\right)=\frac{1}{2} m(r, f)$ and thus

$$
T\left(r, f_{1}\right)=T\left(r, f_{2}\right)=\frac{1}{2} T(r, f)= \begin{cases}r / 2 & (0 \leq r \leq R / 2), \\ r-R / 4 & (R / 2<r<R)\end{cases}
$$

for all $r<R$. Hence,

$$
T\left(r, f_{1} \oplus f_{2}\right)>\max \left\{T\left(r, f_{1}\right), T\left(r, f_{2}\right)\right\} \quad(0<r<R) .
$$




\section{First main theorem}

As usual in the Nevanlinna theory and its tropical analogue in the real line $\mathbf{R}$, the next step from the tropical Poisson-Jensen formula is to formulate the first main theorem. To this end, we will simply modify the notation $L(f):=\inf \left\{f(b): \omega_{f}(b)<\right.$ $0, b \in \mathbf{R}\}$ applied in $\mathbf{R}$ for a tropical meromorphic function $f$ defined in the finite interval $(-R, R)$ as follows:

$$
L_{R}(f):=\inf \left\{f(b): \omega_{f}(b)<0,|b|<R\right\} .
$$

In particular, if $f$ has no poles in $(-R, R)$, then we put $L_{R}(f)=+\infty$ as $\inf \emptyset$. For simplicity, we denote

$$
N(r, f \mid<a):=N(r, f)-N(r, \max (f, a))
$$

with the proviso that $N(r, f \mid<a): \equiv 0$ for $a=-\infty$. Clearly, $N(r, f \mid<a)$ also vanishes identically either when $-\infty<a<L_{R}(f)$ or when $L_{R}(f)=+\infty$. Here we note that $N(r, f \mid<a)$ takes always a non-negative value for any $a \in(-\infty,+\infty)$ and all $r \in[0, R$ ), since it follows by Lemma 3.1 (iv) that

$$
N(r, \max (f, a)) \leq N(r, f)+N(r, a)=N(r, f)
$$

is true for any $a \in(-\infty,+\infty)$ and all $r \in[0, R)$.

Theorem 4.1. Let $f$ be tropical meromorphic in an interval $(-R, R)(0<R \leq$ $\infty)$. Then the asymptotic equality

$$
T\left(r, 1_{\diamond} \oslash(f \oplus a)\right)=T(r, f)-N(r, f \mid<a)-\max (f(0), a)+\varepsilon_{f}(r, a)
$$

hold with a quantity $\varepsilon_{f}(r, a)$ satisfying $0 \leq \varepsilon_{f}(r, a) \leq \max (a, 0)$ for any $a \in[-\infty,+\infty)$ and for all $r \in[0, R)$.

Proof. Making use of the tropical Jensen formula (1.5) to the function $f(x) \oplus a=$ $\max (f(x), a)$ and the definition (4.1), we immediately conclude that our asymptotic equality is equal to

$$
\begin{aligned}
\varepsilon_{f}(r, a) & =T\left(r, 1_{\circ} \oslash(f \oplus a)\right)-T(r, f)+N(r, f \mid<a)+\max (f(0), a) \\
& =T(r, \max (f, a))-T(r, f)+\{N(r, f)-N(r, \max (f, a))\} \\
& =m(r, \max (f, a))-m(r, f) .
\end{aligned}
$$

Applying Lemma 3.1(iv) to the estimate (4.2), we have

$$
\varepsilon_{f}(r, a) \leq m(r, f)+m(r, a)-m(r, f)=m(r, a) \equiv \max (a, 0)
$$

for any $a \in[-\infty,+\infty)$ and for all $r \in[0, R)$.

On the other hand, applying Lemma 3.1(i) to (4.2), we have the lower estimate

$$
\varepsilon_{f}(r, a)=m(r, \max (f, a))-m(r, f) \geq 0
$$

for any $a \in[-\infty,+\infty)$ and for all $r \in[0, R)$. Hence we have obtained the asymptotic equality with this quantity $\varepsilon_{f}(r, a)$ as in the lemma.

We next proceed to prove the following result by a similar way given in [5] as well as in [7] and [10].

Theorem 4.2. The characteristic function $T(r, f)$ is a positive, continuous, nondecreasing piecewise linear function of $r \in[0, R)$. 
Proof. It is immediate to observe first that $T(r, f)$ is positive and continuous, due to the definitions of the proximity and counting functions and to Lemma 1.6. As to the monotonicity of $T(r, f)$, consider first intervals consisting of $r \in[0, R)$ where $f(x)$ does not have a root or a pole at either $x=r$ or $x=-r$. Differentiating the tropical Jensen formula in Corollary 2.2 now results in

$$
f^{\prime}(r)-f^{\prime}(-r)=n(r,-f)-n(r, f) .
$$

Applying the proof of Theorem 3.4 in [5] verbatim, we conclude that $\frac{d}{d r} T(r, f)$ is nonnegative in these intervals, meaning that $T(r, f)$ is non-decreasing therein. Passing over the values of $\pm r$, where $f$ has either a root or a pole, by continuity, the monotonicity of $T(r, f)$ follows. Piecewise linearity of $T(r, f)$ again follows by the definitions of the proximity and counting functions.

Remark. (1) We can prove also this theorem by differentiating the tropical Cartan identity [7, Theorem 3.8]. Indeed,

$$
\begin{aligned}
\frac{d}{d r} T(r, f) & =\frac{d}{d r}\left[\max \left\{N\left(r, 1_{\diamond} \oslash(-f)^{+}\right), N\left(r, 1_{\diamond} \oslash f^{+}\right)\right\}+f(0)^{+}\right] \\
& =\max \left\{n\left(r, 1_{\diamond} \oslash(-f)^{+}\right), n\left(r, 1_{\diamond} \oslash f^{+}\right)\right\} \geq 0
\end{aligned}
$$

holds for all $r \in[0, R)$ off a discrete set. However, the proof of the identity itself requires similar observations as had been used above, (see [7, pp. 73-75]).

(2) Note that $T(r, f)$ is a non-decreasing function of $\log \frac{1}{R-r}$ as well, since

$$
\frac{d}{d r} \log \frac{1}{R-r}=\frac{1}{R-r}>0 .
$$

Similarly as in the real-line case, see [7] and [10], we may now define the order $\rho(f)$, resp. the hyper-order $\rho_{2}(f)$, of a non-constant tropical meromorphic function $f$ in the interval $(-R, R)$. Notice, however, that the growth of $T(r, f)$ in $[0, R)$ should be measured by the unbounded factor $-\log (R-r)$ instead of the variable $r$ itself. Normalizing $-\log (R-r)$ by $\log R-\log (R-r)=\log \frac{R}{R-r}$ which is always positive for $r \in(0, R)$, we introduce the following definitions:

Definition 4.3. Let $f(x)$ be a non-constant tropical meromorphic function in an interval $(-R, R)$. We put

$$
\rho(f):=\limsup _{r \uparrow R} \frac{\log ^{+} T(r, f)}{\log \log \frac{R}{R-r}},
$$

and

$$
\rho_{2}(f):=\limsup _{r \uparrow R} \frac{\log ^{+} \log ^{+} T(r, f)}{\log \log \frac{R}{R-r}} .
$$

Remark. Of course, $\log ^{+}$reduces to $\log$ in $\rho(f)$, whenever $T(r, f) \geq 1$, resp. in $\rho_{2}(f)$, whenever $T(r, f) \geq e$. In this paper, we concentrate in considering tropical meromorphic functions $f$ in $(-R, R)$ satisfying $T(r, f) \uparrow \infty$ as $r \uparrow R$.

Example. If a non-constant tropical meromorphic function $f$ is of rational type in $(-R, R)$, then there are constants $A(>0)$ and $B(\geq 0)$ such that $T(r, f)=A r+B$ for any $r$ sufficiently near $R$ and therefore we then have $\rho(f)=\rho_{2}(f)=0$. Of course, the same conclusion is trivial whenever $T(r, f)$ that is bounded as $r \rightarrow R$. This may happen for a tropical meromorphic function that is not of rational type. As an example, consider a tropical meromorphic function $f$ in $(-R, R)$ which has no poles 
and whose root set is exactly the sequence $a_{n}=R(1-1 / n)(n \in \mathbf{N})$. Assuming $f\left(a_{n+1}\right)-f\left(a_{n}\right)=1 / 2^{n}$ for $n \in \mathbf{N}$ and $f(x) \equiv 0$ for $x<0$, we see that this $f(x)$ is an increasing and convex function in $(-R, R)$ and satisfies

$$
T(r, f)=m(r, f) \leq \sum_{n=1}^{\infty} \frac{1}{2^{n}}=1
$$

\section{Tropical shift in finite intervals}

In the real-line case, the shift $x \rightarrow x+c(c \in \mathbf{R})$ has been applied to obtain the lemma on tropical quotients, see [7, Section 3.4]. In our present finite-interval case, we need to introduce a different shift notion $s(x)$ such that $s(x)$ remains in the same interval for all $x \in(-R, R)$. To this end, first note the formal tropical counter-part to the standard Möbius transformation:

$$
y(t)=(a \otimes t \oplus b) \oslash(c \otimes t \oplus d)=\max (t+a, b)-\max (t+c, d),
$$

where $a, b, c, d \in \mathbf{R}_{-\infty}:=\mathbf{R} \cup\{-\infty\}$ satisfying

$$
a \otimes d \oplus b \otimes c \neq 0 \text { 。 }, \quad \text { i.e. } \quad \max (a+d, b+c) \neq-\infty .
$$

Here we recall the convention

$$
\alpha \otimes 0_{\circ}=0_{\circ} \otimes \alpha=0_{\circ}, \quad \text { i.e. } \quad \alpha+(-\infty)=-\infty+\alpha=-\infty
$$

for $\alpha \in \mathbf{R}_{-\infty}$.

Considering next the closed interval $[-R, R](0<R<\infty)$, and the parameters $a=d=R$ and $b=c=0=1$ 。, we obtain the mapping $\varphi: \mathbf{R} \rightarrow[-R, R]$ with

$$
\begin{aligned}
\varphi(t) & =\left(R \otimes t \oplus 1_{\circ}\right) \oslash(t \oplus R) \\
& =\max (t+R, 0)-\max (t, R) \\
& =R+\max (t,-R)-\max (t, R) \\
& = \begin{cases}-R & (t<-R) \\
t & (-R \leq t \leq R) \\
R & (R<t) .\end{cases}
\end{aligned}
$$

This reduces to the identity mapping on the interval $[-R, R]$, and has the (only) root of multiplicity 1 at $t=-R$ and the (only) pole of multiplicity 1 at $t=R$.

We proceed to a scaling of the mapping $\varphi$ by fixing a constant $\tau \in(0,1)$, and defining two mappings as follows:

$$
s_{\tau}^{+}: \mathbf{R} \rightarrow[-\tau R,(2-\tau) R] \subset \mathbf{R}
$$

and

$$
s_{\tau}^{-}: \mathbf{R} \rightarrow[-(2-\tau) R, \tau R] \subset \mathbf{R}
$$

by

$$
s_{\tau}^{ \pm}(x)=\varphi(\tau x) \pm(1-\tau) R, \quad-\infty<x<\infty .
$$

In more detail, we now have

$$
s_{\tau}^{+}(x)= \begin{cases}-R+(1-\tau) R=-\tau R & \left(x<-\frac{R}{\tau}\right) \\ \tau x+(1-\tau) R=\tau(x-R)+R & \left(-\frac{R}{\tau} \leq x \leq \frac{R}{\tau}\right) \\ R+(1-\tau) R=(2-\tau) R & \left(\frac{R}{\tau}<x\right)\end{cases}
$$


and

$$
s_{\tau}^{-}(x)= \begin{cases}-R-(1-\tau) R=-(2-\tau) R & \left(x<-\frac{R}{\tau}\right) \\ \tau x-(1-\tau) R=\tau(x+R)-R & \left(-\frac{R}{\tau} \leq x \leq \frac{R}{\tau}\right) \\ R-(1-\tau) R=\tau R & \left(\frac{R}{\tau}<x\right) .\end{cases}
$$

The locations and multiplicities of the root, resp. the pole of the original map $\varphi(x)$ are now shifted so that $s_{\tau}^{ \pm}(x)$ has the (only) root of multiplicity $\tau$ at $x=-\frac{R}{\tau}$ and the (only) pole of multiplicity $\tau$ at $x=\frac{R}{\tau}$, both of which being outside of the interval $[-R, R]$ since $\tau \in(0,1)$. Moreover, note that when $s_{\tau}^{ \pm}(x)$ is restricted on $[-R, R]$ we have

$$
\lim _{\tau \downarrow 0} s_{\tau}^{ \pm}(x) \equiv \pm R \quad \text { and } \quad \lim _{\tau \uparrow 1} s_{\tau}^{ \pm}(x) \equiv x .
$$

Also, $s_{\tau}^{+}(x)$, resp. $s_{\tau}^{-}(x)$ has its (unique) fixed point at $x=R$, resp. $x=-R$.

The functions $s_{\tau}^{ \pm}(x)$ possess a number of simple properties required to be a shift in the interval $(-R, R)$. For example, as the counterpart of the property that the $n$th iteration $(x \pm c)^{n}=x \pm n c$ of the shift $x+c(c>0)$ tends monotonely to $\pm \infty$ as $n \rightarrow+\infty$, we observe that the iterations behave like

$$
\left(s_{\tau}^{+}\right)^{n}(x)=s_{\tau^{n}}^{+}(x) \uparrow R \quad \text { and } \quad\left(s_{\tau}^{-}\right)^{n}(x)=s_{\tau^{n}}^{-}(x) \downarrow-R
$$

as $n \rightarrow+\infty$, respectively. We omit listing all those properties up here. Instead, we proceed to combine $s_{\tau}^{+}(x)$ and $s_{\tau}^{-}(x)$ into a unified mapping $s_{\tau}(x)$ by defining

$$
s_{\tau}(x):= \begin{cases}s_{\tau}^{+}(x)=\tau x+(1-\tau) R & \left(\rho_{0}<x<R\right) \\ \tau x+(1-\tau) \frac{R}{\rho_{0}} x & \left(-\rho_{0} \leq x \leq \rho_{0}\right) \\ s_{\tau}^{-}(x)=\tau x-(1-\tau) R & \left(-R<x<-\rho_{0}\right)\end{cases}
$$

for a fixed $\rho_{0} \in(0, R)$. This $s_{\tau}(x)$ is a continuous, monotone increasing mapping of $[-R, R]$ onto itself as one may immediately see. In what follows, fixing a constant $\tau$, we call $s_{\tau}(x)$ as the shift function on the interval $[-R, R]$.

It is elementary to verify that $s_{\tau}(-x)=-s_{\tau}(x)$ on the whole interval $(-R, R)$. Indeed, whenever $-R<-x<-\rho_{0}$, then

$$
s_{\tau}(-x)=s_{\tau}^{-}(-x)=-s_{\tau}^{+}(x)=-s_{\tau}(x),
$$

if $\rho_{0}<-x<R$, then

$$
s_{\tau}(-x)=s_{\tau}^{+}(-x)=-s_{\tau}^{-}(x)=-s_{\tau}(x)
$$

and if $|x| \leq \rho_{0}$, then

$$
s_{\tau}(-x)=\tau(-x)+(1-\tau) \frac{R}{\rho_{0}}(-x)=-\left\{\tau x+(1-\tau) \frac{R}{\rho_{0}} x\right\}=-s_{\tau}(x) .
$$

Finally, observe that the mapping $s_{\tau}(x)$ can naturally be continuously extended outside the interval $(-R, R)$ to satisfy $s_{\tau}(-x)=-s_{\tau}(x)$ on $\mathbf{R}$.

\section{Growth lemma with the shift functions $s_{\tau}^{ \pm}(x)$}

A key goal in this paper is to find a finite interval analogue to the tropical logarithmic quotient lemma in $\mathbf{R}$, see [7, Section 3.4], by means of the shift function $s_{\tau}(x)$ in the finite interval $(-R, R)$. As a preliminary step to this end, we show the following estimates with two mappings $s_{\tau}^{+}(x)$ and $s_{\tau}^{-}(x)$ : 
Lemma 6.1. Let $f$ be a tropical meromorphic function in the interval $(-R, R)$ and let $\tau \in(0,1)$ and $r \in(0, R)$ be constants satisfying

$$
\tau(r-R)+R<\rho
$$

for a given constant $\rho \in(r, R)$. Then for any $x \in[-r, r]$, we have

$$
\begin{aligned}
& \max \left(f\left(s_{\tau}^{ \pm}(x)\right)-f(x), 0\right)=\left(f\left(s_{\tau}^{ \pm}(x)\right)-f(x)\right)^{+} \\
& \leq\left|s_{\tau}^{ \pm}(x)-x\right|\left\{\frac{m(\rho, f)+m(\rho,-f)}{\rho}+n(\rho, f)+n(\rho,-f)\right\} .
\end{aligned}
$$

Proof. Applying the tropical Poisson-Jensen formula (2.1) to $f(t)$ for any $t \in$ $(-\rho, \rho)$, we have

$$
\begin{aligned}
f(t)= & \frac{f(-\rho)+f(\rho)}{2}+\frac{f(\rho)-f(-\rho)}{2 \rho} t \\
& -\frac{1}{2 \rho} \sum_{\left|a_{\mu}\right|<\rho} \tau_{f}\left(a_{\mu}\right)\left(\rho-\max \left(a_{\mu}, t\right)\right)\left(\min \left(a_{\mu}, t\right)+\rho\right) \\
& +\frac{1}{2 \rho} \sum_{\left|b_{\nu}\right|<\rho} \tau_{f}\left(b_{\nu}\right)\left(\rho-\max \left(b_{\nu}, t\right)\right)\left(\min \left(b_{\nu}, t\right)+\rho\right),
\end{aligned}
$$

where we denote by $a_{\mu}$ and $b_{\nu}$ the distinct poles and distinct roots of $f$ in the interval $(-\rho, \rho)$, respectively. Hence we have

$$
\begin{aligned}
f\left(s_{\tau}^{ \pm}(x)\right)-f(x)= & \frac{f(\rho)-f(-\rho)}{2 \rho}\left\{s_{\tau}^{ \pm}(x)-x\right\} \\
& -\frac{1}{2 \rho} \sum_{\left|a_{\mu}\right|<\rho} \tau_{f}\left(a_{\mu}\right)\left\{\left(\left|s_{\tau}^{ \pm}(x)-a_{\mu}\right|-\left|x-a_{\mu}\right|\right) \rho+a_{\mu}\left(s_{\tau}^{ \pm}(x)-x\right)\right\} \\
& +\frac{1}{2 \rho} \sum_{\left|b_{\nu}\right|<\rho} \tau_{f}\left(b_{\nu}\right)\left\{\left(\left|s_{\tau}^{ \pm}(x)-b_{\nu}\right|-\left|x-b_{\nu}\right|\right) \rho+b_{\nu}\left(s_{\tau}^{ \pm}(x)-x\right)\right\},
\end{aligned}
$$

since

$$
\begin{aligned}
& \left(\rho-\max \left(c, t_{1}\right)\right)\left(\min \left(c, t_{1}\right)+\rho\right)-\left(\rho-\max \left(c, t_{2}\right)\right)\left(\min \left(c, t_{2}\right)+\rho\right) \\
& =\left\{\left|t_{2}-c\right|-\left|t_{1}-c\right|\right\} \rho+c\left(t_{2}-t_{1}\right)
\end{aligned}
$$

by the identity (2.2). Noting, moreover, that ||$t_{2}-c|-| t_{1}-c|| \leq\left|t_{2}-t_{1}\right|$, we finally obtain

$$
\begin{aligned}
& \left(f\left(s_{\tau}^{ \pm}(x)\right)-f(x)\right)^{+} \\
& \leq \frac{|f(\rho)-f(-\rho)|}{2 \rho}\left|s_{\tau}^{ \pm}(x)-x\right| \\
& \quad+\frac{1}{2 \rho} \sum_{\left|a_{\mu}\right|<\rho} \tau_{f}\left(a_{\mu}\right) 2 \rho\left|s_{\tau}^{ \pm}(x)-x\right|+\frac{1}{2 \rho} \sum_{\left|b_{\nu}\right|<\rho} \tau_{f}\left(b_{\nu}\right) 2 \rho\left|s_{\tau}^{ \pm}(x)-x\right| \\
& =\left|s_{\tau}^{ \pm}(x)-x\right|\left\{\frac{|f(\rho)-f(-\rho)|}{2 \rho}+\sum_{\left|a_{\mu}\right|<\rho} \tau_{f}\left(a_{\mu}\right)+\sum_{\left|b_{\nu}\right|<\rho} \tau_{f}\left(b_{\nu}\right)\right\}
\end{aligned}
$$




$$
\begin{aligned}
& =\left|s_{\tau}^{ \pm}(x)-x\right|\left\{\frac{f(\rho)^{+}+(-f(-\rho))^{+}+f(-\rho)^{+}+(-f(\rho))^{+}}{2 \rho}+n(\rho, f)+n(\rho,-f)\right\} \\
& =\left|s_{\tau}^{ \pm}(x)-x\right|\left\{\frac{m(\rho, f)+m(\rho,-f)}{\rho}+n(\rho, f)+n(\rho,-f)\right\}
\end{aligned}
$$

as desired.

Remark. Observe that $\left|s_{\tau}^{ \pm}(x)-x\right|=(1-\tau)(R \mp x)$ when $x \in[-R, R]$ by the definition of the functions $s_{\tau}^{ \pm}(x)$ in (5.4) and (5.5).

\section{Lemma on a tropical ratio of logarithmic shift}

As a preparation to a finite-interval version of the tropical quotient lemma, see e.g. [7, Theorem 3.27], we first prove the following lemma which may be understood as a finite-interval counterpart to [4, Lemma 8.3] as well as to [7, Theorem 3.15]:

Lemma 7.1. Let $T:[0, R) \rightarrow[0,+\infty), 0<R<\infty$, be a non-decreasing continuous function of hyper-order

$$
\rho_{2}(T):=\limsup _{r \uparrow R} \frac{\log ^{+} \log ^{+} T(r)}{\log \log \frac{R}{R-r}}<1 .
$$

For any fixed constant $\varepsilon>0$ satisfying $(1+\varepsilon) \rho_{2}(T)<1$ and for a constant $p(\varepsilon)$ such that $(1+\varepsilon) \rho_{2}(T)<p(\varepsilon)<1$, we put

$$
\varphi_{\varepsilon}(r):=\left(\log \frac{R}{R-r}\right)^{-\frac{1-p(\varepsilon)}{1+\varepsilon}} \quad(0 \leq r<R) .
$$

Then we have

$$
T\left(s_{\tau}(r)\right)<T(r)+\varphi_{\varepsilon}(r) T(r)
$$

where $r$ tends to $R$ outside of a set $F \subset\left[\rho_{0}, R\right)$ of measure

$$
\int_{F} d \log \log \frac{R}{R-r}=\int_{F} \frac{d r}{(R-r) \log \frac{R}{R-r}}<+\infty .
$$

Proof. First, denote

$$
\nu(r)=\left\{1+\varphi_{\varepsilon}(r)\right\}^{-1}=\left\{1+\left(\log \frac{R}{R-r}\right)^{-\frac{1-p(\varepsilon)}{1+\varepsilon}}\right\}^{-1}
$$

for $r \in\left[\rho_{0}, R\right)$ and assume, contrary to the assertion, that the set $F$ of $r$ with

$$
T(r) \leq \nu(r) T\left(s_{\tau}(r)\right)
$$

is of infinite measure, $\int_{F} d \log \log \frac{R}{R-r}=\infty$. Let $r_{0}\left(\geq \rho_{0}\right)$ be the smallest element in the set $F$. Define then a sequence $\left\{r_{n}\right\}_{n \in \mathbf{N}}$ inductively by

$$
r_{n}:=\min \left\{r \in F \cap\left[s_{\tau}\left(r_{n-1}\right), R\right)\right\}
$$

for each $n \in \mathbf{N}$. Observe that $s_{\tau}\left(r_{n-1}\right)>r_{n-1}$ for each $n \in \mathbf{N}$; this can easily be seen by the definition of $s_{\tau}(x)$ in (5.6). The sequence $\left\{r_{n}\right\}_{n \geq 0}$ satisfies

- $r_{n} \geq s_{\tau}\left(r_{n-1}\right)$ for all $n \in \mathbf{N}$,

- $F \subset \bigcup_{n=0}^{\infty}\left[r_{n}, s_{\tau}\left(r_{n}\right)\right]$, and

- $T\left(r_{n}\right) \leq \nu\left(r_{n}\right) T\left(r_{n+1}\right)$ for all $n \in \mathbf{N} \cup\{0\}$. 
Therefore,

$$
\int_{F} d \log \log \frac{R}{R-r} \leq \sum_{n=1}^{\infty} \int_{r_{n}}^{s_{\tau}\left(r_{n}\right)} d \log \log \frac{R}{R-r}+O(1) .
$$

For each $n \in \mathbf{N}$, we now obtain

$$
\begin{aligned}
\int_{r_{n}}^{s_{\tau}\left(r_{n}\right)} d \log \log \frac{R}{R-r} & =\left[\log \log \frac{R}{R-r}\right]_{r_{n}}^{s_{\tau}\left(r_{n}\right)}=\log \left\{\frac{\log \frac{R}{R-s_{\tau}\left(r_{n}\right)}}{\log \frac{R}{R-r_{n}}}\right\} \\
& =\log \left\{\frac{\log \frac{\tau\left(R-r_{n}\right)}{R}}{\log \frac{R-r_{n}}{R}}\right\}=\log \left\{1+\frac{\log (1 / \tau)}{\log \frac{R}{R-r_{n}}}\right\} .
\end{aligned}
$$

Assume now, for a while, that

$$
R-r_{n} \leq R \exp \left(-n^{1+\varepsilon}\right)
$$

thus

$$
\log \frac{R}{R-r_{n}} \geq n^{1+\varepsilon}
$$

is true for each $n \in \mathbf{N}$. Then

$$
\int_{r_{n}}^{s_{\tau}\left(r_{n}\right)} d \log \log \frac{R}{R-r} \leq \log \left\{1+\frac{\log (1 / \tau)}{n^{1+\varepsilon}}\right\} \leq \frac{\log (1 / \tau)}{n^{1+\varepsilon}}
$$

for every $n \in \mathbf{N}$, so that

$$
\begin{aligned}
\int_{F} d \log \log \frac{R}{R-r} & \leq \sum_{n=1}^{\infty} \frac{\log (1 / \tau)}{n^{1+\varepsilon}}+O(1) \leq\{\log (1 / \tau)\} \int_{1}^{\infty} \frac{d x}{x^{1+\varepsilon}}+O(1) \\
& =\frac{1}{\varepsilon} \log \frac{1}{\tau}+O(1)<\infty
\end{aligned}
$$

a contradiction. Therefore, the sequence $\left\{r_{n}\right\}_{n \in \mathbf{N}}$ has a subsequence, $\left\{r_{n_{j}}\right\}_{j \in \mathbf{N}}$, such that we have

$$
R-r_{n_{j}}>R \exp \left(-n_{j}{ }^{1+\varepsilon}\right), \quad \text { hence } \quad \log \frac{R}{R-r_{n_{j}}}<n_{j}{ }^{1+\varepsilon}
$$

for each $j \in \mathbf{N}$. Since

$$
T\left(r_{1}\right) \leq \nu\left(r_{1}\right) T\left(r_{2}\right) \leq \cdots \leq\left\{\prod_{k=1}^{n_{j}-1} \nu\left(r_{k}\right)\right\} T\left(r_{n_{j}}\right)
$$

and thus

$$
\begin{aligned}
\log T\left(r_{n_{j}}\right) & \geq \log T\left(r_{1}\right)-\sum_{k=1}^{n_{j}-1} \log \nu\left(r_{k}\right) \\
& =\log T\left(r_{1}\right)+\sum_{k=1}^{n_{j}-1} \log \left\{1+\varphi_{\varepsilon}\left(r_{k}\right)\right\} \\
& \geq \log T\left(r_{1}\right)+\left(n_{j}-1\right) \log \left\{1+\varphi_{\varepsilon}\left(r_{n_{j}}\right)\right\}
\end{aligned}
$$


for each $j \in \mathbf{N}$. Therefore,

$$
\begin{aligned}
\rho_{2}(T) & \geq \limsup _{j \rightarrow \infty} \frac{\log ^{+} \log ^{+} T\left(r_{n_{j}}\right)}{\log \log \frac{R}{R-r_{n_{j}}}} \\
& \geq \limsup _{j \rightarrow \infty} \frac{\log \left\{\left(n_{j}-1\right) \varphi_{\varepsilon}\left(r_{n_{j}}\right) \log \left\{1+\varphi_{\varepsilon}\left(r_{n_{j}}\right)\right\}^{1 / \varphi_{\varepsilon}\left(r_{n_{j}}\right)}\right\}}{\log \log \frac{R}{R-r_{n_{j}}}} \\
& \geq \limsup _{j \rightarrow \infty}\left\{\frac{\log \left(n_{j}-1\right)}{\log \log \frac{R}{R-r_{n_{j}}}}-\frac{1-p(\varepsilon)}{1+\varepsilon} \cdot \frac{\log \log \frac{R}{R-r_{n_{j}}}}{\log \log \frac{R}{R-r_{n_{j}}}}\right\} \\
& \geq \frac{1}{1+\varepsilon}-\frac{1-p(\varepsilon)}{1+\varepsilon}=\frac{p(\varepsilon)}{1+\varepsilon}>\rho_{2}(T),
\end{aligned}
$$

which is a contradiction, completing the proof of this lemma.

We are now ready to give a finite-interval version of the tropical quotient lemma in the following form:

Theorem 7.2. Let $f$ be a tropical meromorphic function of hyper-order $\rho_{2}(f)<$ 1 in an open interval $(-R, R)$ and $\tau$ be a fixed constant with $\tau \in(0,1)$. Let then $\varepsilon$ be an arbitrary positive constant such that $(1+\varepsilon) \rho_{2}(f)<1$, and take a constant $\delta$ satisfying $(1+\varepsilon) \rho_{2}(f)<\delta<1$. Then, for the constant $\eta$ given by

$$
\eta=\frac{1-\delta}{(1+\varepsilon)\left(\rho_{2}(f)+\varepsilon\right)}>0
$$

we have

$$
\frac{m\left(r, f\left(s_{\tau}(x)\right) \oslash f(x)\right)}{T(r, f)}=O\left\{\left(\log ^{+} T(r, f)\right)^{-\eta}\right\}+O(R-r),
$$

where $r$ tends to $R$ outside a set of $F \subset\left[\rho_{0}, R\right)$ such that

$$
\int_{F} d \log \log \frac{R}{R-r}=\int_{F} \frac{d r}{(R-r) \log \frac{R}{R-r}}<\infty .
$$

Proof. We divide the subsequent proof in two parts, for the convenience of the reader.

(1) Take first a fixed $r_{0} \in\left[\rho_{0}, R\right)$ such that $T\left(r_{0}, f\right) \geq f(0)$. This may be done, since otherwise $T\left(\rho_{0}, f\right)<f(0)$ and so

$$
m(0, f)=f(0)^{+} \leq T(0, f) \leq T\left(\rho_{0}, f\right)<f(0),
$$

a contradiction.

Take next two constants $\kappa$ and $\lambda$ with $\kappa<\lambda<\tau<1$ and put

$$
\rho=s_{\lambda}(r)=\lambda(r-R)+R \in\left(r_{0}, R\right)
$$

for a value of $r \in\left(r_{0}, R\right)$. By (5.6),

$$
s_{\kappa}(x)>s_{\lambda}(r)>s_{\tau}(r) \quad\left(r_{0} \leq r<R\right) .
$$

By the application of Lemma 6.1, we have

$$
\begin{aligned}
& m\left(r, f\left(s_{\tau}(x)\right) \oslash f(x)\right) \\
& \leq(1-\tau)(R-r)\left\{\frac{m(\rho, f)+m(\rho,-f)}{\rho}+n(\rho, f)+n(\rho,-f)\right\} .
\end{aligned}
$$


Since

$$
m(\rho, f)+m(\rho,-f) \leq T(\rho, f)+T(\rho,-f)=2 T(\rho, f)+f(0) \leq 3 T(\rho, f)
$$

by the tropical Jensen formula, it follows by the monotonicity of $T(r, f)$ that

$$
(1-\tau)(R-r) \cdot \frac{m(\rho, f)+m(\rho,-f)}{\rho} \leq \frac{3(1-\tau)}{r_{0}}(R-r) T\left(s_{\kappa}(r), f\right)
$$

when $r>r_{0}$. The previous Lemma 7.1 shows

$$
T\left(s_{\kappa}(r), f\right) \leq\{1+o(1)\} T(r, f)
$$

where $o(1)$ stands for a quantity that approaches to zero as $r \uparrow R$ outside a set of $F$ as in the statement of our theorem, see Lemma 7.1.

(2) The second part of this proof is devoted to giving a similar estimate

$$
(1-\tau)(R-r)\{n(\rho, f)+n(\rho,-f)\}=O\left\{\left(\log ^{+} T(r, f)\right)^{-\eta}\right\} T(r, f)
$$

again outside of the set $F$. Recall that the integrated counting functions are nonnegative and non-decreasing. Moreover, $f(x)$ is of hyper-order $\rho_{2}(f)<1$, hence

$$
\limsup _{r \uparrow R} \frac{\log ^{+} \log ^{+} N(r, \pm f)}{\log \log \frac{R}{R-r}} \leq \rho_{2}(f)
$$

by the tropical Jensen formula. Applying now Lemma 7.1 to $N(r, \pm f)$, we obtain

$$
N\left(s_{\kappa}(r), f\right)+N\left(s_{\kappa}(r),-f\right)-N(\rho, f)-N(\rho,-f) \leq \varphi_{\varepsilon}(\rho)\{N(\rho, f)+N(\rho,-f)\}
$$

outside a set $F$ of $r$ in $\left[\rho_{0}, R\right)$ that satisfies $\int_{F} d \log \log \frac{R}{R-r}<\infty$. Here we put $\rho=$ $s_{\lambda}(r)$ and

$$
\varphi_{\varepsilon}(\rho)=\left(\log \frac{R}{R-\rho}\right)^{-\frac{1-\delta}{1+\varepsilon}}
$$

Then we observe

$$
\begin{aligned}
N\left(s_{\kappa}(r), \pm f\right)-N(\rho, \pm f) & \geq \frac{1}{2} \int_{\rho}^{s_{\kappa}(r)} n(t, \pm f) d t \geq \frac{1}{2} n(\rho, \pm f)\left(s_{\kappa}(r)-\rho\right) \\
& =\frac{1}{2}(\lambda-\kappa)(R-r) n(\rho, \pm f),
\end{aligned}
$$

so that we obtain

$$
\begin{aligned}
& (1-\tau)(R-r)\{n(\rho, f)+n(\rho,-f)\} \\
& \leq \frac{2(1-\tau)}{\lambda-\kappa}\left\{N\left(s_{\kappa}(r), f\right)+N\left(s_{\kappa}(r),-f\right)-N(\rho, f)-N(\rho,-f)\right\} \\
& \leq \frac{2(1-\tau)}{\lambda-\kappa} \varphi_{\epsilon}(\rho)\{N(\rho, f)+N(\rho,-f)\} \\
& \leq \frac{6(1-\tau)}{\lambda-\kappa} \varphi_{\varepsilon}(r) T\left(s_{\kappa}(r), f\right)
\end{aligned}
$$

for any $r \in\left(r_{0}, R\right) \backslash F$, provided that $r$ is close enough to $R$. Here we have used the elementary observation that

$$
\varphi_{\varepsilon}(\rho)=\left(\log \frac{R}{\lambda(R-r)}\right)^{-\frac{1-\delta}{1+\varepsilon}}<\left(\log \frac{R}{R-r}\right)^{-\frac{1-\delta}{1+\varepsilon}}=\varphi_{\varepsilon}(r) .
$$


Furthermore, there exists $r_{1} \in\left[r_{0}, R\right)$ such that

$$
\log \log ^{+} T(r, f)<\left(\rho_{2}(f)+\varepsilon\right) \log \log \frac{R}{R-r},
$$

hence $\left(\log ^{+} T(r, f)\right)^{1 /\left(\rho_{2}(f)+\varepsilon\right)}<\log \frac{R}{R-r}$ is true for all $r \in\left[r_{1}, R\right)$. This shows that

$$
\varphi_{\varepsilon}(r)<\left(\log ^{+} T(r, f)\right)^{-\frac{1-\delta}{(1+\varepsilon)\left(\rho_{2}(f)+\varepsilon\right)}} .
$$

We now recall $\eta>0$, see Theorem 7.2:

$$
\eta=\frac{1-\delta}{(1+\varepsilon)\left(\rho_{2}(f)+\varepsilon\right)} .
$$

Thus our estimate may be written as

$$
(1-\tau)(R-r)\{n(\rho, f)+n(\rho,-f)\} \leq \frac{6(1-\tau)}{\lambda-\kappa}\left(\log ^{+} T(r, f)\right)^{-\eta} T\left(s_{\kappa}(r), f\right)
$$

outside of the set $F$. Another application of Lemma 7.1 shows

$$
T\left(s_{\kappa}(r), f\right)=\{1+o(1)\} T(r, f)
$$

and thus (7.3) holds outside an exceptional set which can be merged into the $F$.

By the equation (7.3) together with equations (7.1) and (7.2), we are done.

\section{Second main theorem}

We have now proved that an $s_{\tau}$-operator analogue of the logarithmic derivative lemma of the form

$$
\lim _{r \uparrow R} \frac{m\left(r, f\left(s_{\tau}(x)\right) \oslash f(x)\right)}{T(r, f)}=0
$$

outside an exceptional set $F$ given as above. Here we need of course to assume $T(r, f) \rightarrow \infty$ as $r \uparrow R$. Hence, for such functions, that is, tropical meromorphic functions $f:(-R, R) \rightarrow \mathbf{R}$ with unbounded characteristic function $T(r, f)$, we can also deduce an $s_{\tau}$-operator analogue of the second main theorem by tracing verbatim the reasoning for non-constant tropical meromorphic functions $f: \mathbf{R} \rightarrow \mathbf{R}$ in [7] and [10]. This is mainly because that the Nevanlinna functions $m(r, f), N(r, f)$ and $T(r, f)$ in $\mathbf{R}$ and in $(-R, R)$ have the common properties as we have seen in this note. Indeed, one finds the following correspondence of the results in [7] and those in this note:

- As variants of the lemma on tropical quotients, Theorem 3.27 of [7, p. 82] corresponds to our Theorem 7.2;

- Lemmas 3.28, 3.29, 3.30 and Remark 3.21 of [7, pp. 83-85] as well as Proposition 3.34 of $[7$, p. 87] are all independent of the domains $\mathbf{R}$ and $(-R, R)$;

- The statements and the proofs of Propositions 3.32 and 3.33 of [7, pp. 86-87] are also available still in this note, if one replaces $f(x+c)$ by $f\left(s_{\tau}(x)\right)$ and $-f(c)$ by $-f\left(s_{\tau}(0)\right)=-f(0)$ all at once;

- Propositions 3.36, 3.37 and 3.38 as well as Remark 3.39 of [7, pp. 87-90] are also true in this note with the replacement of the notation $L_{f}$ appeared in the latter two statements by our $L_{R}(f)$ introduced in Section 4;

- The statement and the proof of Theorem 3.40 of [7, pp. 90-92] are available in this note again by replacing $f(x+c)$ by $f\left(s_{\tau}(x)\right)$ and $-f(c)$ by $-f(0)$ all at once. 
After all, we have the following

Theorem 8.1. Let $f$ be a tropical meromorphic function in an open interval $(-R, R)$ and let $\tau \in(0,1), q \in \mathbf{N}$ and $a_{1}, \ldots, a_{q} \in \mathbf{R}$ be distinct such that $\max \left\{a_{1}, \ldots, a_{q}\right\}<L_{R}(f)$. Then

$$
\begin{aligned}
q T(r, f) \leq & \sum_{j=1}^{q} N\left(r, 1_{\circ} \oslash\left(f \oplus a_{j}\right)\right)+T\left(r, f\left(s_{\tau}(x)\right)\right)-N\left(r, 1_{\diamond} \oslash f\left(s_{\tau}(x)\right)\right) \\
& +m\left(r, f\left(s_{\tau}(x)\right) \oslash f(x)\right)-f(0)+q \max \left\{a_{1}, \ldots, a_{q}, 0\right\} \\
& +(q-1) \max \left\{-a_{1}, \ldots,-a_{q}, 0\right\}+\sum_{j=1}^{q} \max \left\{f(0), a_{j}\right\} .
\end{aligned}
$$

Theorem 7.2 states $m\left(r, f\left(s_{\tau}(x)\right) \oslash f(x)\right)$ is small in the sense there with respect to $T(r, f)$ under those assumptions. Here noting that $\left|s_{\tau}(x)\right| \leq s_{\tau}(r)$ whenever $|x| \leq r \in[0, R)$, we have

$$
V\left(r, g\left(s_{\tau}(x)\right)\right) \leq V\left(s_{\tau}(r), g\right)
$$

both with $V=T$ and $g=f$ and with $V=N$ and $g=1 \diamond \oslash f$. Further, the application of Lemma 7.1 in each case deduces

$$
V\left(s_{\tau}(r), g\right) \leq V(r, g)+\left\{O\left(\left(\log ^{+} T(t, f)\right)^{-\eta}\right)+O(R-r)\right\} T(r, f)
$$

outside an exceptional set $F$ in the sense of Lemma 7.1 as well as of Theorem 7.2. Then we can now prove our second main theorem as the counterpart of Theorem 3.41 of $[7$, p. 92].

Theorem 8.2. Suppose $f$ is a tropical meromorphic function of hyper-order $\rho_{2}(f)<1$ in an open interval $(-R, R)$ and its characteristic function is unbounded, and let $a_{1}, \ldots, a_{q}, q \geq 1$ be distinct real values that satisfy $\max \left\{a_{1}, \ldots, a_{q}\right\}<L_{R}(f)$. Let $\varepsilon$ be an arbitrary positive constant such that $(1+\varepsilon) \rho_{2}(f)<1$. If we take a constant $\delta$ satisfying $(1+\varepsilon) \rho_{2}(f)<\delta<1$ and put $\eta=\frac{1-\delta}{(1+\varepsilon)\left(\rho_{2}(f)+\varepsilon\right)}(>0)$, then

$$
\begin{aligned}
(q-1) T(r, f) \leq & \sum_{j=1}^{q} N\left(r, 1_{\circ} \oslash\left(f \oplus a_{j}\right)\right)-N\left(r, 1_{\diamond} \oslash f\right) \\
& +\left\{O\left(\left(\log ^{+} T(t, f)\right)^{-\eta}\right)+O(R-r)\right\} T(r, f),
\end{aligned}
$$

as $r \uparrow R$ outside a set of $F \subset[0, R)$ such that $\int_{F} d \log \log \frac{R}{R-r}<\infty$.

\section{Discussion}

We are listing here a few natural problems that should indeed be settled in the present finite interval setting of tropical meromorphic functions to obtain a satisfactory consideration.

(1) One is first to ask whether the assumption that $\rho_{2}(f)<1$ is necessary to obtain a result similar to Theorem 7.2.

(2) The second problem is to ask whether it is possible to construct a continuous piecewise linear function in $(-R, R)$ which behaves like a tropical exponential function $e_{\alpha}(x)(\alpha \neq 0, \pm 1)$ in $(-\infty, \infty)$, see [7, Section 1.2.4]. Actually, one might expect that such a function $y(x)$ could be easily constructed in $[0, r)$ by fixing its roots at $\rho_{0}, \rho_{1}, \ldots$, where $\rho_{n+1}=s_{\tau}\left(\rho_{n}\right)=\tau \rho_{n}+(1-\tau) R$, and 
letting its slope increase to infinity as $n \rightarrow \infty$. Defining now $\tilde{y}(x)$ so that $\tilde{y}(2 x-R)=y(x)$ for $0<x<R$, we get a tropical meromorphic function of exponential type in $(-R, R)$. However, this function is not as expected, since for $-R<t<-\rho_{0}$ we have $\tilde{y}(t)=y((R+t) / 2)$, while $\tilde{y}\left(s_{\tau}(t)\right)=$ $y\left(\left(R+s_{\tau}(t)\right) / 2\right)=y(\tau(R+t) / 2)$, but $s_{\tau}((R+t) / 2)-\tau(R+t) / 2=(1-\tau) R \neq 0$. This means that $\tilde{y}(x)$ does not satisfy in $\left(-R,-\rho_{0}\right)$ a functional equation similar to $f(x+1)=f(x)^{\otimes \alpha}$ satisfied by the exponential function $e_{\alpha}(x)$ in $\mathbf{R}$.

(3) Finally, the problem related to the preceding one is whether functional equations of type $y\left(s_{\tau}(x)\right)=y(x)^{\alpha}$ permit tropical meromorphic solutions in $(-R, R)$, or at least for $|x|>\rho_{0}$ being sufficiently close to $R$. Also, provided such tropical meromorphic solutions exists, what about their order, respectively hyper-order? As to this problem, see the next example below.

(4) The previous problem (3) may also be asked for the case when $\alpha=1$, that is, for the ' $s_{\tau}$-periodic' functions in the sense that they satisfy the relation $y\left(s_{\tau}(x)\right)=y(x)$ for $|x|>\rho_{0}$. As to this problem, see the final remark.

Example. For the sake of simplicity, we take $R=1$ and $\rho_{0}=\tau=\frac{1}{2}$, respectively. Then by definition we have

$$
s(x):=s_{1 / 2}(x)= \begin{cases}\frac{1}{2} x-\frac{1}{2} & \left(-1<x<-\frac{1}{2}\right) \\ \frac{3}{2} x & \left(-\frac{1}{2} \leq x \leq \frac{1}{2}\right) \\ \frac{1}{2} x+\frac{1}{2} & \left(\frac{1}{2}<x<1\right) .\end{cases}
$$

As above, we have $s(x)=-s(-x)$ and $s(x) \in\left[\frac{1}{2}, 1\right)$ whenever $x \in\left[\frac{1}{3}, 1\right)$. Hence we will first restrict our attention on the interval $\left[\frac{1}{3}, 1\right)$ and find a solution $y(x)$ of the equation

$$
y(s(x))=\alpha y(x), \quad y(1 / 3)=1,
$$

there for a real constant $\alpha \neq 0, \pm 1$. Of course, after finding such solution $y(x)$, our desired tropical meromorphic functions, say $\varepsilon_{\alpha}(x)$, on $(-1,1)$ should be defined as follows:

$$
\varepsilon_{\alpha}(x)= \begin{cases}-y(x) & \left(-1<x \leq-\frac{1}{3}\right) \\ 3 x & \left(-\frac{1}{3} \leq x \leq \frac{1}{3}\right) \\ y(x) & \left(\frac{1}{3}<x<1\right)\end{cases}
$$

Then $\varepsilon_{\alpha}(s(x))=\alpha \varepsilon_{\alpha}(x)$ when $\frac{1}{3} \leq|x|<1$. On the other hand, this does not satisfy the functional equation in the interval $\left(-\frac{1}{3}, \frac{1}{3}\right)$.

However, we proceed by looking at the orbit of the point $x=1 / 3$ by $s(x)$. Inductively, we have

$$
\begin{aligned}
s\left(\frac{1}{3}\right) & =\frac{3}{2} \cdot \frac{1}{3}=\frac{1}{2} \\
s^{2}\left(\frac{1}{3}\right) & =s\left(\frac{1}{2}\right)=\frac{1}{2} \cdot\left(\frac{1}{2}+1\right)=\frac{2+1}{2^{2}} \\
s^{3}\left(\frac{1}{3}\right) & =s\left(\frac{2+1}{2^{2}}\right)=\frac{1}{2} \cdot\left(\frac{2+1}{2^{2}}+1\right)=\frac{2^{2}+2+1}{2^{3}}, \\
& \vdots \\
s^{n}\left(\frac{1}{3}\right) & =\frac{2^{n-1}+2 n-2+\cdots+2+1}{2^{n}}=\frac{2^{n}-1}{2^{n}}=1-\frac{1}{2^{n}}
\end{aligned}
$$


for $n \geq 1$. In fact,

$$
s^{n+1}\left(\frac{1}{3}\right)=s\left(1-\frac{1}{2^{n}}\right)=\frac{1}{2}\left(1-\frac{1}{2^{n}}+1\right)=1-\frac{1}{2^{n+1}}
$$

holds.

Then we have required $y(x)$ to satisfy $y(1 / 3)=1$ and thus have

$$
y\left(\frac{1}{2}\right)=y\left(s\left(\frac{1}{3}\right)\right)=\alpha y\left(\frac{1}{3}\right)=\alpha .
$$

Further, we inductively obtain

$$
y\left(s^{n}\left(\frac{1}{2}\right)\right)=\alpha y\left(s^{n-1}\left(\frac{1}{3}\right)\right)=\cdots=\alpha^{n-1} y\left(s\left(\frac{1}{3}\right)\right)=\alpha^{n}
$$

for any $n \in \mathbf{N}$.

On the closed interval $\left[s^{n}\left(\frac{1}{3}\right), s^{n+1}\left(\frac{1}{3}\right)\right](n \in \mathbf{N})$, let us define

$$
\begin{aligned}
y(x) & =\frac{\alpha^{n+1}-\alpha^{n}}{s^{n+1}(1 / 3)-s^{n}(1 / 3)}\left(x-s^{n}(1 / 3)\right)+\alpha^{n} \\
& =\frac{\alpha^{n+1}-\alpha^{n}}{\frac{2^{n+1}-1}{2^{n+1}}-\frac{2^{n}-1}{2^{n}}}\left(x-\frac{2^{n}-1}{2^{n}}\right)+\alpha^{n} \\
& =2^{n+1} \alpha^{n}(\alpha-1) x-2\left(2^{n}-1\right) \alpha^{n+1}+\left(2^{n+1}-1\right) \alpha^{n} .
\end{aligned}
$$

Since $s(x)=\frac{1}{2} x+\frac{1}{2} \in\left[s^{n-1}\left(\frac{1}{3}\right), s^{n}\left(\frac{1}{3}\right)\right]$ if $\in\left[s^{n-1}\left(\frac{1}{3}\right), s^{n}\left(\frac{1}{3}\right)\right]$, we have

$$
\begin{aligned}
y(s(x)) & =2^{n+1} \alpha^{n}(\alpha-1) s(x)-2\left(2^{n}-1\right) \alpha^{n+1}+\left(2^{n+1}-1\right) \alpha^{n} \\
& =2^{n} \alpha^{n}(\alpha-1) x+2^{n} \alpha^{n}(\alpha-1)-2\left(2^{n}-1\right) \alpha^{n+1}+\left(2^{n+1}-1\right) \alpha^{n} \\
& =\alpha\left\{2^{n} \alpha^{n-1}(\alpha-1) x-2\left(2^{n-1}-1\right) \alpha^{n}+\left(2^{n}-1\right) \alpha^{n-1}\right\}=\alpha y(x)
\end{aligned}
$$

for each $n \in \mathbf{N}$, so that $y(s(x))=\alpha y(x)$ whenever $x \in[1 / 3,1)$.

Assume $\alpha>1$ and therefore $\varepsilon_{\alpha}(x)$ has no poles at all so that we have $T\left(r, \varepsilon_{\alpha}\right)=$ $m\left(r, \varepsilon_{\alpha}\right)$. Since the solution $y(x)>0$ only when $x>0$, we further have $m\left(r, \varepsilon_{\alpha}\right)=$ $\frac{1}{2} \varepsilon_{\alpha}(r)$. For any $r \in\left[\frac{1}{3}, 1\right)$, there exists an $n \in \mathbf{N}$ such that

$$
s^{n}\left(\frac{1}{3}\right) \leq r<s^{n+1}\left(\frac{1}{3}\right), \quad \text { that is, } \quad \frac{1}{2^{n}} \geq 1-r>\frac{1}{2^{n+1}},
$$

so that

$$
n \leq \log _{2} \frac{1}{1-r}<n+1
$$

holds. Then $\alpha^{n} \leq \varepsilon_{\alpha}(r)<\alpha^{n+1}$, that is, $\frac{1}{2} \alpha^{n} \leq T\left(r, \varepsilon_{\alpha}\right)<\frac{1}{2} \alpha^{n+1}$, which implies

$$
n \log \alpha-\log 2 \leq \log T\left(r, \varepsilon_{\alpha}\right)<(n+1) \log \alpha-\log 2,
$$

and thus

$$
\log n+\log \log \alpha \leq \log \left(\log T\left(r, \varepsilon_{\alpha}\right)+\log 2\right)<\log (n+1)+\log \log \alpha .
$$

Since we know

$$
\log n \leq \log \log \frac{1}{1-r}-\log \log 2<\log (n+1),
$$

it follows altogether

$$
\frac{\log n}{\log (n+1)}+o(1) \leq \frac{\log \log T\left(r, \varepsilon_{\alpha}\right)}{\log \log \frac{1}{1-r}} \leq \frac{\log (n+1)}{\log n}+o(1)
$$


as $n \rightarrow \infty$, that is,

as we expected.

$$
\lim _{r \uparrow 1} \frac{\log \log T\left(r, \varepsilon_{\alpha}\right)}{\log \log \frac{1}{1-r}}=1,
$$

On the other hand,

$$
y(s(x))-y(x)=(\alpha-1) y(x)
$$

implies the estimate

$$
m\left(r, \varepsilon_{\alpha}(s(x)) \oslash \varepsilon_{\alpha}(x)\right)=T(r, \varepsilon)+O(1) .
$$

Remark. We finally add a brief remark on $s_{\tau}(x)$-periodic functions. Given a continuous piecewise linear function $\pi(x)$ on the interval $[1 / 3,1 / 2]$, define a function $y(x)$ on $[1 / 2,1)$ so that for a given point $x \in\left[s^{n}(1 / 3), s^{n+1}(1 / 2)\right], n \in \mathbf{N}, y(x):=$ $\pi\left(s^{-n}(x)\right)$. This now implies $y(s(x))=\pi\left(s^{-n}(x)\right)=y(x)$. We next extend this $y(x)$ over $(-1,1)$ as an even function over $(-1,-1 / 3] \cup[1 / 3,1)$, and as a constant in $[-1 / 3,1 / 3]$. The periodicity in $[1 / 2,1)$ implies that there are two constants $c_{1}, c_{2}$ greater than one such that both $1 / c_{1} \leq m(r, y)<c_{1}$ and $1 / c_{2} \leq n(r, y)<c_{2}$ hold for all $r \in(0,1)$. By a similar discussion as in the preceding example, we deduce that $\log T(r, y)$ is comparable with $\log \log \frac{1}{1-r}$ as $r \uparrow 1$. Thus, the growth is regular and the order is equal to one, deviating from the case when $\alpha \neq 1$. A completely similar discussion could be made for the case $\alpha=-1$, that is, for the anti- $s_{\tau}(x)$-periodic case.

\section{References}

[1] Cherry, W., and Z. Ye: Nevanlinna's theory of value distribution. - Springer-Verlag, Berlin, 2001.

[2] Halburd, R. G., and R. Korhonen: Difference analogue of the lemma of the logarithmic derivative with applications to difference equations. - J. Math. Anal. Appl. 314, 2006, 477-487.

[3] Halburd, R. G., and R. Korhonen: Finite-order meromorphic solutions and the discrete Painlevé equations. - Proc. London Math. Soc. 94, 2007, 443-474.

[4] Halburd, R. G., R. Korhonen, and K. Tohge: Holomorphic curves with shift-invariant hyperplane preimages. - Trans. Amer. Math. Soc. 366, 2014, 4267-4298.

[5] Halburd, R. G., and N. Southall: Tropical Nevanlinna theory and ultra-discrete equations. - Int. Math. Res. Not. IMRN 2009, 2009, 887-911.

[6] Hayman, W. K.: Meromorphic functions. - Clarendon Press, Oxford, 1964.

[7] Korhonen, R., I. Laine, and K. Tohge: Tropical value distribution theory and ultra-discrete equations. - World Scientific Publishing Company, 2015.

[8] Korhonen, R., and K. Tohge: Second main theorem in the tropical projective space. - Adv. Math. 298, 2016, 693-725.

[9] Laine, I.: Nevanlinna theory and complex differential equations. - Walter de Gruyter, BerlinNew York, 1993.

[10] Laine, I., and K. Tohge: Tropical Nevanlinna theory and second main theorem. - Proc. Lond. Math. Soc. 102, 2011, 883-922.

[11] Speyer, D., and B. Sturmfels: Tropical mathematics. - Math. Mag. 82:3, 2009, 163-173. 